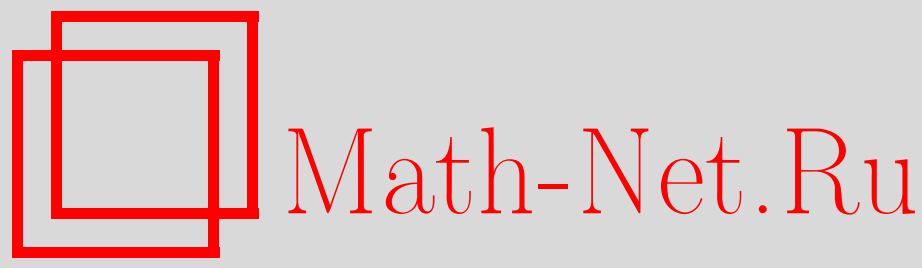

Л. Мартина, Динамика некоммутативного монополя, TMФ, 2012, том 172, номер 2, 285-295

DOI: https://doi.org/10.4213/tmf6958

Использование Общероссийского математического портала Math-Net.Ru подразумевает, что вы прочитали и согласны с пользовательским соглашением http://www . mathnet.ru/rus/agreement

Параметры загрузки:

IP : 54.157 .27 .8

26 апреля 2023 г., 09:38:55

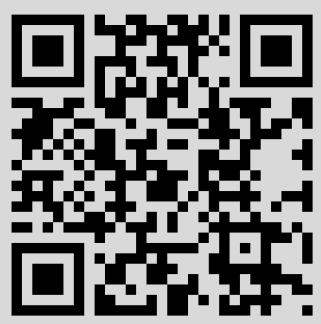




\title{
ДИНАМИКА НЕКОММУТАТИВНОГО МОНОПОЛЯ
}

\begin{abstract}
Рассмотрена электрически заряженная частица, одновременно взаимодействующая с магнитным монополем и с дуальным монополем в пространстве импульсов. Эта система представляет собой прототип трехмерной системы, содержащей некоммутирующие и/или неканонические переменные, но обладающей геометрической, равно как и калибровочной симметриями в координатном и импульсном пространствах. Обсуждаются основные свойства движений и законов сохранения и аналогия со случаем плоского движения.
\end{abstract}

Ключевые слова: некоммутативная система, монополь, заряженная частица.

\section{1. ВВЕДЕНИЕ}

В последнее десятилетие значительно вырос интерес к определенным классам динамических систем, как классических, так и квантовых, в которых коммутационные соотношения между операторами координат не равны нулю. Это предположение приписывается самому Гейзенбергу и подразумевает введение фундаментальной длины, которая может контролировать сингулярности, возникающие при малых расстояниях в квантовой теории поля, таким же образом, как постоянная Планка контролирует такие сингулярности в классическом фазовом пространстве. Математические основы этого подхода были заложены в так называемой "некоммутативной геометрии", развитой в семидесятые годы Конном [1] (см. также работу [2], содержащую обзор современного состояния дел в этой области). Заметим, однако, что некоммутативность координат в рамках нерелятивистского подхода впервые появилась в работе [3], посвященной теории электропроводности в сильном внешнем магнитном поле. Поскольку при этом электронные состояния сосредоточены на низшем уровне Ландау, получается эффективное описание в терминах некоммутирующих операторов координат центров частиц. Эта процедура имеет классический аналог, формализованный в работе [4] путем использования так называемой “экзотической" механической модели на двумерной плоскости (см. также работу [5] и замечания к ней в [6]). Эта модель задается 1-формой Картана [7]

$$
\lambda=\left(p_{i}-A_{i}\right) d x_{i}-\left(\frac{\vec{p}^{2}}{2 m}+e V\right) d t+\frac{\theta}{2} \epsilon_{i j} p_{i} d p_{j},
$$

*Dipartimento di Fisica, Università del Salento Sezione INFN di Lecce, Lecce, Italy. E-mail: martina@le.infn.it 
содержащей компоненты вектор-потенциала $A_{i}(\vec{r})$, электрический потенциал $V(\vec{r})$ (для простоты будем предполагать, что оба потенциала не зависят от времени) и постоянную $\theta$, называемую параметром некоммутативности. Ввиду присутствия последнего члена выражение (1) отличается от стандартной формулы

$$
\lambda=\frac{\partial L}{\partial p_{i}} d x^{i}+\left(L-\frac{\partial L}{\partial p_{i}} p_{i}\right) d t .
$$

Тем не менее в подходящих локальных координатах $\xi^{\alpha}$ оно задает локальную лагранжеву функцию $\mathcal{L}=a_{\alpha}\left(\xi^{\beta}\right) \dot{\xi}^{\alpha}$ первого порядка такую, что

$$
\int_{\widetilde{\gamma} \subset \mathcal{U}} \lambda=\int_{\gamma \subset \pi \mathcal{U}} \mathcal{L} d t
$$

есть функционал действия вдоль поднятой мировой линии $\widetilde{\gamma}=(\gamma(t), \dot{\gamma}(t), t)$ в эволюционном пространстве и $\gamma \subset \pi \mathcal{U}$ является проекцией в конфигурационном пространстве.

Отметим, что величина $\lambda$ зависит от калибровки, в отличие от 2-формы Лагранжа-Сурье [7] $\sigma=d \lambda$, и получим выражение

$$
\sigma=d p_{i} \wedge d x_{i}+\theta d p_{1} \wedge d p_{2}+e B d x_{1} \wedge d x_{2}-\left(\frac{p_{i} d p_{i}}{m}-e E_{i}\right) \wedge d t,
$$

в которое входят только калибровочно-инвариантная компонента магнитного поля $B(\vec{r})=\epsilon_{i j} \partial_{i} A_{j}$, перпендикулярная к плоскости, и компонента электрического поля $E_{i}=-\partial_{i} V(\vec{r})$, лежащая в плоскости.

Введем эбфективную массу $m^{*}=m(1-e \theta B)$. При $m^{*} \neq 0$ 2-форму (2) можно расщепить на гамильтонову форму $\sigma=\omega-d H \wedge d t$, где $\omega$ замкнута, и регулярную 2-форму на фазовом пространстве $T Q$. При этом $H$ оказывается гамильтонианом на $T Q \times \mathbb{R}$ со скобкой Пуассона

$$
\left\{x_{1}, x_{2}\right\}=\frac{m}{m^{*}} \theta, \quad\left\{x_{i}, p_{j}\right\}=\frac{m}{m^{*}} \delta_{i j}, \quad\left\{p_{1}, p_{2}\right\}=\frac{m}{m^{*}} e B,
$$

которые удовлетворяют тождеству Якоби при любом поле В. Уравнения движения, имеющие вид

$$
m^{*} \dot{x}_{i}=p_{i}-e m \theta \epsilon_{i j} E_{j}, \quad m^{*} \dot{p}_{i}=e E_{i}+e B \epsilon_{i j} \dot{x}_{j},
$$

отличаются от стандартных следующими свойствами:

1) они содержат член аномальной скорости $-e m \theta \epsilon_{i j} E_{j}$, так что $\dot{\vec{r}} \sharp \vec{p}$;

2) производная импульса $\vec{p}$ по-прежнему определяется силой Лоренца;

3) возникает взаимодействие между $\theta$ и полем $B$ в $m^{*}$.

При аномальной массе, равной нулю, т. е. в случае, когда магнитное поле принимает критическое значение $B_{\text {crit }}=1 / e \theta$, система становится сингулярной. При этом процедура симплектической редукции [8] приводит к двумерной системе, обладающей замечательной пуассоновой структурой $\left\{x_{1}, x_{2}\right\}=\theta$. Таким образом, симплектическая плоскость играет одновременно роль конфигурационного и фазового пространств. Единственными движениями будут движения, отвечающие закону Холла $p_{i}=\varepsilon_{i j} E_{j} / B_{\text {crit. }}$. Более того, при квантовании редуцированной системы 
не только перестают коммутировать операторы координат частиц, но и квантовые уравнения движения приводят к появлению волновых функций Лавлина [9], являющихся вакуумными состояниями дробного квантового эффекта Холла. В результате можно утверждать, что классическими аналогами анионов будут на самом деле экзотические частицы системы (4).

В обзорной статье [6] обсуждались некоторые примеры двумерных моделей, обобщающих форму (2) и уравнения (4). Здесь напомним, что пуассонову структуру (3) можно получить, применяя ли-алгебраический метод Кириллова-Костанта-Сурье построения динамических систем, которые обладают галилеевой группой $(2+1)$-движений, снабженной двукратным центральным расширением [10], [11]. Двумя когомологическими параметрами при этом оказываются обычная масса $m$ и "экзотический" параметр $\theta$, описывающий степень некоммутативности генераторов галилеевых бустов, $\left\{K_{1}, K_{2}\right\}=-\theta m^{2}$. В контексте физики конденсированного состояния параметр $\theta$ отождествляется с постоянной кривизной Берри, порождаемой решеточной структурой; он действует на электронные волновые пакеты [12], [13]. Этот параметр не является константой, а зависит от квазиимпульса кристалла. С другой стороны, его также можно рассматривать как "нерелятивистскую тень спина" релятивистской частицы, если применить так называемую свертку Джакива-Наира [14]. Основной результат состоит в том, что последний член в выражении (1) при этом можно заменить на зависящую от $\vec{p}$ подходящую 1 -форму $\mathcal{R}_{j}(\vec{r}, \vec{p}) d p_{j}$, которая задает удобную кривизну в импульсном пространстве.

\section{2. ОБЩАЯ МОДЕЛЬ В РАЗМЕРНОСТИ $3+1$}

Недавно в работе [15] при поиске обобщений подхода, описанного в работах [13], [16]-[18], было доказано, что 2-форму Лагранжа-Сурье в размерности три можно построить как внешнюю производную 1-формы Картана:

$$
\lambda=(\vec{p}-e \overrightarrow{\mathcal{A}}) \cdot d \vec{r}-\overrightarrow{\mathcal{R}} \cdot d \vec{p}-\mathcal{T} d t,
$$

где поле $\overrightarrow{\mathcal{A}}(\vec{r}, t)$ представляет собой обычный потенциал электромагнитного поля, так что $\vec{B}=\nabla_{\vec{r}} \times \overrightarrow{\mathcal{A}}$. Электрическое поле (а на самом деле любое поле, определяемое скалярным потенциалом) задается формулой $\vec{E}=-\nabla_{\vec{r}} \mathcal{T} / e+\partial_{t} \overrightarrow{\mathcal{A}}$, где предполагается, что $\mathcal{T}(\vec{r}, \vec{p}, t)=\mathcal{E}(\vec{p}, t)+e \varphi(\vec{r}, t)$, и постулируется, что поле $\vec{E}$ никаким образом не зависит от $\vec{p}$. С другой стороны, новый тип калибровочного поля $\overrightarrow{\mathcal{R}}(\vec{r}, \vec{p}, t)$ задает так называемое "дуальное магнитное поле" $\vec{\kappa}=\nabla_{\vec{p}} \times \overrightarrow{\mathcal{R}}$. Более правильно будет рассмотреть вклад, пропорциональный $d p_{i} \wedge d p_{j}$, в 2-форму Лагранжа-Сурье

$$
\begin{aligned}
\sigma=d \lambda= & {\left[\left(1+\mu_{i i}\right) d p_{i}-e E_{i} d t\right] \wedge\left(d r_{i}-g_{i} d t\right)+\frac{1}{2} e B_{k} \epsilon_{k i j} d r_{i} \wedge d r_{j}+} \\
& +\frac{1}{2} \kappa_{k} \epsilon_{k i j} d p_{i} \wedge d p_{j}-\mu_{i j} d r_{i} \wedge d p_{j},
\end{aligned}
$$

где введены флуктуации локальной массы $\mu_{i j}=\partial_{r_{i}} \mathcal{R}_{j}$ и поток массы

$$
g_{i}=\frac{\partial_{p_{i}} \mathcal{T}-\partial_{t} \mathcal{R}_{i}}{1+\mu_{i i}} .
$$

Формула (6) обобщает формулу (12.46) из работы [7] и считается основным законом механики, а ее дифференциальная форма в силу тождества $d \sigma \equiv 0$ называется принципом Максвелла для силовых полей. 
Таким образом, не только электромагнитное поле, порождаемое потенциалом $\overrightarrow{\mathcal{A}}$, должно удовлетворять однородным уравнениям Максвелла; аналогичные соотношения должны выполняться для “дуальных электромагнитных полей” $\kappa_{k}$ и $g_{i}$, задаваемых потенциалом $\overrightarrow{\mathcal{R}}$ в переменных $p_{i}, t$, а компоненты $\mu_{i j}$ должны удовлетворять дополнительным однородным линейным соотношениям относительно переменных $r_{i}$, $p_{j}, t[15]$. Если вторая группа когомологий на полном эволюционном пространстве нетривиальна, то в общем случае не существует глобальных потенциалов вида $\overrightarrow{\mathcal{A}}$ или $\overrightarrow{\mathcal{R}}$. Тем самым полное эволюционное пространство необходимо расщепить на несколько карт и определить 1-форму Картана (5) локально на каждой из них. С другой стороны, форма Лагранжа-Сурье будет определена глобально, и если к тому же выполняется соотношение $\partial_{t} \overrightarrow{\mathcal{A}}=\partial_{t} \overrightarrow{\mathcal{R}} \equiv 0$, то становится возможным расщепить форму (6) и представить ее в гамильтоновом виде:

$$
\sigma=\omega-d \mathcal{T} \wedge d t
$$

Симплектическая 2-форма $\omega$ на фазовом пространстве $T Q=\{\xi=(\vec{r}, \vec{p})\}$ принимает матричный вид:

$$
\omega_{\alpha \beta}=\left(\begin{array}{cc}
\mathbf{0} & \mathbf{1} \\
\mathbf{1} & -\left(\epsilon_{k i j} \kappa_{k}\right)
\end{array}\right)\left(\begin{array}{cc}
\mathbf{1}+\boldsymbol{\Psi} & \mathbf{0} \\
\mathbf{0} & \mathbf{1}
\end{array}\right)\left(\begin{array}{cc}
\mathbf{1}+\boldsymbol{\mu} & \mathbf{0} \\
\left(e \epsilon_{k i j} B_{k}\right) & -(\mathbf{1}+\boldsymbol{\mu})
\end{array}\right),
$$

где $\boldsymbol{\Psi}=\left[\left(\epsilon_{k i j} \kappa_{k}\right)(\mathbf{1}+\boldsymbol{\mu})^{-1}\left(e \epsilon_{k i j} B_{k}\right)+\boldsymbol{\mu}^{\mathrm{T}}-\boldsymbol{\mu}\right](\mathbf{1}+\boldsymbol{\mu})^{-1}-$ косимплектическая матрица, в терминах которой можно выписать уравнения Гамильтона.

Однако в общем случае глобально определенные канонические переменные Дарбу нельзя найти ни в явном, ни в неявном виде. Если присутствует только электромагнитный потенциал $\overrightarrow{\mathcal{A}}$ или, наоборот, только потенциал $\overrightarrow{\mathcal{R}}$, то можно произвести локальные точечные преобразования (вида $p_{i}^{\prime}=p_{i}-\mathcal{A}_{i}(\vec{r})$ или $r_{i}^{\prime}=r_{i}-\mathcal{R}_{i}(\vec{p})$ соответственно), приводящие к канонически сопряженным наборам переменных. В отличие от этого случая, если все переменные $r_{i}$ и $p_{i}$ становятся некоммутирующими, то не известно никакой явной формулы, задающей глобальные переменные Дарбу, кроме случая, когда $B_{k}, E_{k}, \kappa_{k}$ постоянные и $g_{k} \equiv p_{k}$ [4]. В результате для систем, взаимодействующих с внешними полями, следует использовать коммутативные или, наоборот, некоммутативные переменные, если они появляются в потенциалах, таким образом, они становятся настоящими наблюдаемыми для данной модели. В этой перспективе следует отметить, что приведенная выше процедура задания взаимодействия между механической системой, содержащей некоммутативные переменные, и электромагнитным полем (схема минимального добавления) существенно отличается от стандартной процедуры минимальной связи $p_{i} \rightarrow p_{i}-e A_{i}$, поскольку последняя предполагает наличие нетривиальной замены пуассоновой структуры по определению формы $\omega$ в выражениях (7), (8).

В действительности в размерности два и для постоянных $\kappa_{3} \equiv \theta$ точечные преобразования $x_{i}=\hat{x}_{i}+e \theta \epsilon_{i j} \hat{A}_{j}\left(\hat{x}_{1}, \hat{x}_{2}, t\right), p_{i}=\hat{p}_{i}-e \hat{A}_{i}\left(\hat{x}_{1}, \hat{x}_{2}, t\right)$ задают отображение между двумя формулировками с помощью классического преобразования Зайберга-Виттена между сопровождающими электромагнитными полями [19]. В частности, это преобразование связывает между собой магнитные поля (потенциалы):

$$
\widehat{B}\left(\hat{x}_{1}, \hat{x}_{2}, t\right)=\frac{B\left(x_{1}, x_{2}, t\right)}{1-e \theta B\left(x_{1}, x_{2}, t\right)},
$$


где величины со шляпками удовлетворяют соотношениям пуассоновой структуры

$$
\left\{\hat{x}_{1}, \hat{x}_{2}\right\}=\theta, \quad\left\{\hat{x}_{i}, \hat{p}_{j}\right\}=\delta_{i j}, \quad\left\{\hat{p}_{1}, \hat{p}_{2}\right\}=e \widehat{B} .
$$

Тем не менее до сих пор не получено никаких результатов в размерности три для непостоянного дуального магнитного поля.

Отметим наконец, что калибровочная свобода, присутствующая в поле $\overrightarrow{\mathcal{R}}$, зависящем от переменных $p_{i}$, приводит к тому, что понятие координаты становится калибровочно-неинвариантным, и мы не можем выписать явные формулы, кроме случая постоянных силовых полей. Далее мы приведем конкретный пример такой структуры. Напомним здесь только то, что элемент объема фазового пространства задается формулой [15]

$$
\sqrt{\operatorname{det}\left(\omega_{\alpha \beta}\right)}=\left|\operatorname{det}(1+\mu)-e \vec{B}^{\mathrm{T}}(1+\mu) \vec{\kappa}\right| \neq 0 .
$$

Такой множитель обобщает знаменатели, присутствующие в скобках Пуассона (3), и его обращение в ноль свидетельствует о редуцировании фазового пространства.

Следует также заметить, что пуассонова структура, равно как и формы $\sigma$ и $\omega$, зависят только от калибровочно-инвариантных переменных, а координаты $r_{i}$ оказываются в общем случае некоммутативными относительно скобки Пуассона. Некоторые примеры систем, которые удается записать в рамках приведенного выше формализма, собраны в работе [6]. Далее мы обсуждаем модель дубля монополей.

\section{3. ДУБЛЬ МОНОПОЛЕЙ}

Концепция монополя в пространстве импульсов $\vec{\kappa}=\frac{\theta \vec{p}}{|\vec{p}|^{3}}$ была впервые введена в работе [7] и проананализирована в [20] с целью дать описание безмассовой релятивистской частицы с ненулевой спиральностью (фотона). Эта концепция может рассматриваться как дуальная по отношению к более известной теории монополя Дирака. Она оказывается согласованной с экспериментальными данными, полученными при наблюдении аномального эффекта Холла [21] и спинового эффекта Холла [22]. Движение заряженной частицы в таком поле обсуждалось с теоретической точки зрения в работах [17] и [23].

Таким образом, естественно рассмотреть одновременно магнитный монополь Дирака и дуальный монополь, восстанавливая тем самым "симметрию", потерянную в модели импульсного монополя, описанного выше. Более того, можно также получить конкретную реализацию такой модели на экспериментальных данных о существовании изолированных монопольных возбуждений в спиновых решетках типа спинового льда [24] или на основе предложения работы [25] в несколько ином контексте. Чтобы включить в рассмотрение оба явления, необходимо найти подходящее поведение фазы Берри, приобретаемой волновым пакетом электрона при прохождении через решетку, как описано в работе [12]. Для краткости будем называть такую систему дублем монополей.

Косимплектическая структура, без труда получаемая из выражения (8), записывается как

$$
\omega^{\alpha, \beta}=\frac{1}{M^{*}}\left(\begin{array}{cc}
\theta|\vec{r}|^{3} \tilde{p} & -|\vec{r}|^{3}|\vec{p}|^{3} \mathbf{1}-e \theta(\vec{p} \otimes \vec{r})^{\mathrm{T}} \\
|\vec{r}|^{3}|\vec{p}|^{3} \mathbf{1}+e \theta \vec{p} \otimes \vec{r} & e|\vec{p}|^{3} \tilde{r}
\end{array}\right),
$$


где через $е$ обозначается константа связи между зарядом и магнитным монополем, $\tilde{p}_{i j}=\varepsilon_{i j k} p_{k}, \tilde{r}_{i j}=\varepsilon_{i j k} r_{k}$ и эффективная масса имеет вид

$$
M^{*}=|\vec{r}|^{3}|\vec{p}|^{3}-e \theta \vec{r} \cdot \vec{p} .
$$

Из формы (9) получим $\sqrt{\operatorname{det}\left(\omega^{\alpha, \beta}\right)}=|\vec{r}|^{3}|\vec{p}|^{3} / M^{*}$. Тогда условие равенства нулю эффективной массы $M^{*}$ налагает неголономные связи на динамику модели.

Предположим, что никаких других полей нет. Тогда для заряженной частицы с единичной массой в дубле монополей свободный гамильтониан записывается как $H=|\vec{p}|^{2} / 2$, и уравнения движения имеют вид

$$
\begin{aligned}
M^{*} \dot{r}_{i} & =\left(p_{i}-e \theta \frac{r_{i}}{|\vec{p}||\vec{r}|^{3}}\right)|\vec{r}|^{3}|\vec{p}|^{3}, \\
M^{*} \dot{p}_{i} & =e \varepsilon_{i j k} p_{j} r_{k}|\vec{p}|^{3} .
\end{aligned}
$$

Если задаться вопросом поиска законов сохранения, отличных от закона сохранения энергии, который задается гамильтонианом $H$, то мы придем к вектору

$$
\vec{j}=\vec{r} \times \vec{p}-\frac{\theta}{|\vec{p}|} \vec{p}-\frac{e}{|\vec{r}|} \vec{r},
$$

который всегда имеет компоненты на плоскости $(\vec{r}, \vec{p})$. Эти компоненты удовлетворяют коммутационным соотношениям $\left\{j_{i}, j_{j}\right\}=\varepsilon_{i j k} j_{k}$ и коммутируют с гамильтонианом относительно пуассоновой структуры (9). Тогда вектор $\vec{j}$ можно отождествить с полным угловым моментом, включающим в себя механическую, электромагнитную и дуальную составляющие и принимающим обычный вид в случае, когда имеется только монополь Дирака или только дуальный монополь при $\theta \rightarrow 0$ или при $e \rightarrow 0$ соответственно, как это показано в работах [17], [26].

Поскольку $H, \vec{j}^{2}$ и $j_{z}$ коммутируют и независимы, можно заключить, что система дубля монополей представляет собой интегрируемую модель. Однако нарушение тождеств Якоби

$$
\frac{1}{2} \varepsilon_{i j k}\left[p_{i},\left[p_{j}, p_{k}\right]\right]=e \delta(\vec{r}), \quad \frac{1}{2} \varepsilon_{i j k}\left[r_{i},\left[r_{j}, r_{k}\right]\right]=\theta \delta(\vec{p})
$$

делает это заключение сомнительным. Более того, это нарушение связано с нетривиальной топологической структурой фазового пространства $(\vec{r}, \vec{p}$,$) , вторая группа$ когомологий которого равна $\mathbb{R} \otimes \mathbb{R} \otimes \mathbb{R} \otimes \mathbb{R}$. Отсюда можно заключить, что построение расслоений над этим базовым пространством нетривиально.

Более того, в силу геометрического построения монополей Дирака в данном случае необходимо иметь четыре различных локальных набора координат, на которых можно регулярным образом определить 1 -формы $(\overrightarrow{\mathcal{A}}, \overrightarrow{\mathcal{R}})$. На пересечении двух таких наборов координат 1-формы должны быть связаны локальными калибровочными преобразованиями, которые задаются двумя независимыми функциями. Это соответствует построению главного $(U(1) \otimes U(1))$-расслоения над $S^{2} \times S^{2}$. Чтобы полученная при этом структура представляла собой многообразие, слои должны переходить в слои после обхода по любому замкнутому пути. Это приводит к появлению квантования как магнитного заряда, так и дуального заряда в целых числах с множителем 1/2: $e=N_{\mathrm{m}} / 2$ и $\theta=N_{\mathrm{d}} / 2$ [17]. 
Отсюда получаем, что

$$
|\vec{j}|^{2}=(\vec{r} \times \vec{p})^{2}+(|\theta|+|e|)^{2}-2|e \theta|(1-\operatorname{sgn}(e \theta) \cos (\widehat{\vec{r} \vec{p}})),
$$

таким образом, вектор $|\vec{j}|$ имеет ненулевой минимум, который достигается, в частности, при $\vec{p} \| \vec{r}$. Более того, разлагая вектор $\vec{r}$ на параллельную и ортогональную к $\vec{p}$ компоненты, получим, что $|\vec{j}|$ не зависит от угла между ортогональной компонентой и направлением оси координат.

Что касается хорошо изученной системы электрический заряд-магнитный монополь, то минимальный полный угловой момент учитывает вклады как от электромагнитного, так и от дуального взаимодействия. Но условие сохранения полного углового момента налагает ограничения на возможные движения, которые могут происходить только вдоль определенных траекторий, пересекающих пятимерное неограниченное подмногообразие фазового пространства, задаваемое условием равенства нулю эффективной массы: $\mathcal{M}=\left\{(\vec{r}, \vec{p}): M^{*}=0\right\}$.

Заметим, что сама по себе скобка Пуассона $\left\{M^{*}, H\right\}$ не равна нулю, но при сужении ее на $\mathcal{M}$ мы получаем

$$
\left.\left\{M^{*}, H\right\}\right|_{\mathcal{M}}=3|\vec{p}|^{2}\left(|\vec{r}|^{4}|\vec{p}|^{4}-e^{2} \theta^{2}\right) \vec{p} \cdot \vec{r} .
$$

Обращение в ноль скобки (13) вместе с массой $M^{*}$ согласуется с условием параллельности

$$
p_{i}=\operatorname{sgn}(e \theta) \sqrt{|e \theta|} \frac{r_{i}}{|\vec{r}|^{2}},
$$

предписанным уравнениями движения (11), (12). В этом пределе уравнение (12) удовлетворяется тождественно, а уравнение (11) принимает вид

$$
\dot{r}_{i}=\operatorname{sgn}(e \theta) \sqrt{|e \theta|} \frac{r_{i}}{|\vec{r}|^{2}},
$$

поскольку скорость и линейный импульс теперь пропорциональны друг другу (на самом деле равны, если масса равна единице).

Выбрав произвольные начальные данные $\left\{\vec{r}_{0}, \vec{p}_{0}\right\}$ такие, что $\left|\vec{j}_{0}\right|=|\theta|+|e|$, получим, что существует радиус-вектор $\vec{r}_{\text {сr }}$ с направлением

$$
\frac{\vec{r}_{\mathrm{cr}}}{\left|\vec{r}_{\mathrm{cr}}\right|}=-\operatorname{sgn}(e) \frac{\vec{j}_{0}}{|\theta|+|e|},
$$

для которого связи (10) и (14) совпадают в первый раз. Поскольку в этом случае гамильтониан принимает вид

$$
\left.H\right|_{\mathcal{M}}=\frac{|e \theta|}{2\left|\vec{r}_{\mathrm{cr}}\right|^{2}}=E_{0},
$$

где $E_{0}-$ его значение в начальной точке, можно заключить, что $\left|\vec{r}_{\mathrm{cr}}\right|=\sqrt{|e \theta| / 2 E_{0}}$, или

$$
\vec{r}_{\mathrm{cr}}=-\operatorname{sgn}(e) \frac{\sqrt{|e \theta|}}{|\theta|+|e|} \frac{\vec{j}_{0}}{\sqrt{2 E_{0}}} .
$$




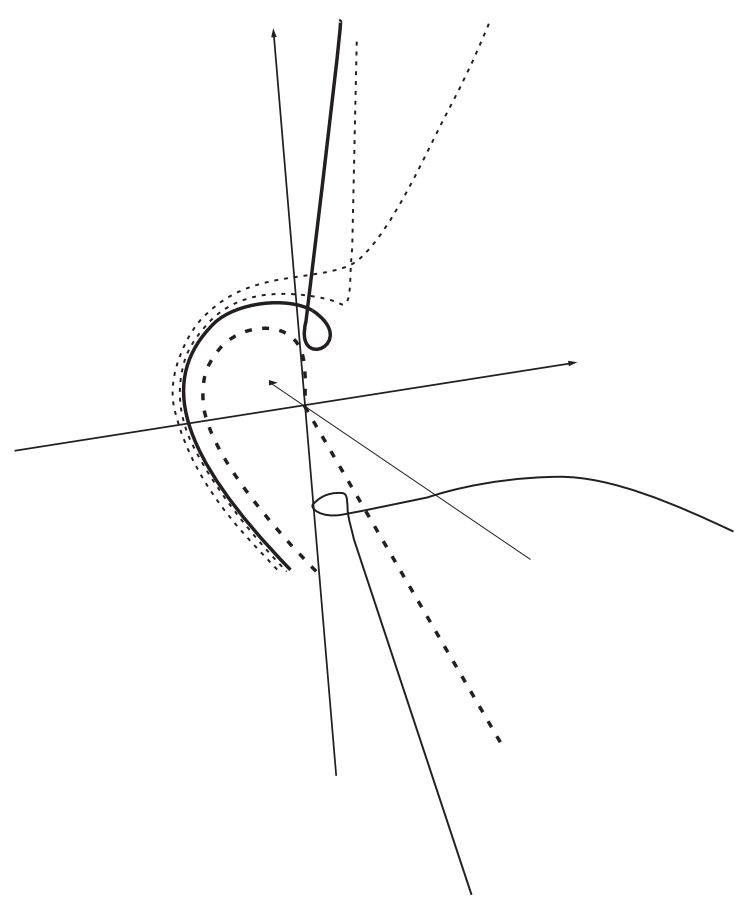

Рис. 1. Крупный план картины рассеяния пяти зарядов с равными энергиями, сталкивающихся на дубле монополей; начальные точки всех зарядов лежат в одной плоскости на $-\infty$ на горизонтальной оси, выходящей из страницы. Для зарядов различны только угловые моменты: жирная сплошная кривая отвечает критическому режиму $|\vec{j}|=|\theta|+|e|$; для сравнения приведены две другие изначально близкие траектории с бо́льшим угловым моментом (пунктир); жирная штриховая кривая отвечает частице с угловым моментом $\vec{j}$, удовлетворяющим неравенству ||$\theta|-| e||<|\vec{j}|<|\theta|+|e|$. Жирные кривые пересекаются в точке с импульсом, отвечающим $M^{*}=0$. С этого момента они становятся прямыми линиями. Наконец, тонкая сплошная кривая обладает тем же критическим угловым моментом $|\vec{j}|=|\theta|+|e|$, но начинается из симметрично расположенной точки.

Поставляя полученное выражение в (14), (15), получим импульс и скорость частицы,

$$
\dot{\vec{r}}_{\mathrm{cr}}=\sqrt{2 E_{0}} \frac{\vec{r}_{\mathrm{cr}}}{\left|\vec{r}_{\mathrm{cr}}\right|}
$$

движение которой отныне будет подчиняться динамике со связями.

На самом деле на инвариантном критическом подмногообразии $\mathcal{M}$ симплектическая 2-форма $\omega$ приводится к виду

$$
\left.\omega\right|_{\mathcal{M}}=\frac{1}{2} \varepsilon_{i j k} \frac{e}{|\vec{r}|^{3}}\left(1+\left|\frac{\theta}{e}\right|\right) r_{k} d r_{i} \wedge d r_{j}
$$


и пропорциональна с точностью до множителя $e /|\vec{r}|^{3}$ симплектической 2-форме, индуцированной на $s o(3)^{*}$ присоединенным действием группы $S O(3)$. Если ограничиться обычным расслоением над сферой с индуцированными скобками Пуассона

$$
\{\vartheta, \varphi\}=-\left[|e|\left(\left|\frac{\theta}{e}\right|+1\right)|\vec{r}|^{2} \sin \varphi\right]^{-1}
$$

для угловых переменных, то мы приходим к тривиальным уравнениям движения $\dot{\vartheta}=\dot{\varphi}=0$, поскольку гамильтониан зависит только от радиальной переменной. Тогда координаты $\vartheta, \varphi$ фиксируются выбором направления $\vec{r}_{\mathrm{cr}} /\left|\vec{r}_{\mathrm{cr}}\right|$, а радиус-вектор возрастает линейно в соответствии с формулой

$$
\vec{r}=\left(\frac{\sqrt{2 E_{0}} t}{\left|\vec{r}_{\mathrm{cr}}\right|}+1\right) \vec{r}_{\mathrm{cr}}
$$

где время $t$ отсчитывается от момента контакта с подмногообразием нулевой эффективной массы.

В случае начальных данных, удовлетворяющих неравенству

$$
|| \theta|-| e||<|\vec{j}|<|\theta|+|e|,
$$

численные расчеты свидетельствуют о сходном поведении, но аналитические вычисления являются, хотя и аналогичными, но более сложными.

Результаты расчета рассеяния пяти зарядов с равными энергиями и различными угловыми моментами, которые сталкиваются на дубле монополей, приведены на рис. 1.

\section{4. ЗАКЛЮЧЕНИЕ}

Можно получить обширный класс динамических систем, если постулировать 1-форму Картана вида (1) в размерности три, приводящую к 2-форме ЛагранжаСурье (2). Обобщение на случай большего числа степеней свободы, как нам представляется, можно провести непосредственно. Мы привели условия, обеспечивающие возможность гамильтоновой формулировки таких систем. С помощью этих условий можно проанализировать свойства интегрируемости систем с помощью стандартных методов. Мы расширили концепцию калибровочной симметрии как на случай стандартных электромагнитных потенциалов, так и на случай "дуальных" потенциалов, зависящих от импульсных переменных. В этой связи мы вкратце обсудили роль канонических переменных, но этот вопрос требует дальнейших исследований. В качестве поучительного примера мы рассмотрели систему дубля монополей, в которой сохраняется энергия и угловой момент. Предложена постановка эксперимента для наблюдения конкретной реализации такой модели. Описано явление обращения в ноль эффективной массы, которое приводит к определенным ограничениям движения до движений Холла на плоскости (см. раздел 1) с очень похожим механизмом, основанным на взаимодействии между магнитным полем и его дуальным партнером.

Однако необходимо прояснить детали редуцированных движений, в частности для угловых моментов, удовлетворяющих неравенству ||$\theta|-| e||<|\vec{j}|<|\theta|+|e|$. Эти результаты пока не нашли своего отражения в исследованиях рассеяния зарядов на 
магнитном монополе, и поэтому можно предположить, что дифференциальные поперечные сечения рассеяния будут сильно зависеть от параметров задачи. Алгебра симметрий дубля монополей есть $s o(3) \times \mathbb{R}$, но не известно, существует ли в этой системе вектор типа Рунге-Ленца (который присутствует в других монополеподобных системах). В этой связи может быть полезным введение тонко настроенного потенциала, содержащего член с обратным квадратом расстояния и ньютоновский член, как было предложено Макинтошем, Циснеросом и Цванцигером некоторое время назад, по аналогии с результатами, полученными в работе [26]. Наконец, в духе работ [27] модель дубля монополей, предложенная в настоящей работе, может быть полезной при обобщении соответствий между системами типа заряд-монополь и спиновыми частицами или анионами.

Благодарности. Автор выражает признательность П. Хорвати, М. Плющаю и П. Штихелю за постановку задачи и постоянные стимулирующие обсуждения. Работа частично финансово поддержана Sezione INFN di Lecce (проект LE41). Paбота представляет собой расширенную версию доклада, сделанного на конференции "Solitons in $1+1$ and $2+1$ dimensions, DS, KP and all that" в честь семидесятилетия профессоров М. Бойти и $Ф$. Пемпинелли.

\section{Список литературы}

[1] A. Connes, Noncommutative Geometry, Academic Press, San Diego, CA, 1994; A. Connes, M. R. Douglas, A. Schwarz, JHEP, 02 (1998), 003, 35 pp., arXiv: hep-th/9711162.

[2] P. Aschieri, H. Grosse, G. Landi, R. Szabo (eds.), SIGMA, 6 (2010), Special issue on Noncommutative Spaces and Fields.

[3] R. Peierls, Z. Phys., 80:11-12 (1933), 763-791.

[4] C. Duval, P. A. Horváthy, Phys. Lett. B, 479:1-3 (2000), 284-290, arXiv: hep-th/0002233; J. Phys. A, 34:47 (2001), 10097-10107, arXiv: hep-th/0106089.

[5] V.P. Nair, A.P. Polychronakos, Phys. Lett. B, 505:1-4 (2001), 267-274, arXiv: hep-th/0011172.

[6] P. A. Horváthy, L. Martina, P. C. Stichel, SIGMA, 6 (2010), 060, 26 pp.

[7] J.-M. Souriau, Structure des systèmes dynamiques, Dunod, Paris, 1970.

[8] R. Abraham, J. Marsden, Foundations of Mechanics, Benjamin, Reading, MA, 1978.

[9] R. B. Laughlin, Phys. Rev. Lett., 50:18 (1983), 1395-1398.

[10] D. R. Grigore, J. Math. Phys., 37:1 (1996), 460-473, arXiv: hep-th/9312048.

[11] A. Ballesteros, M. Gadella, M. A. del Olmo, J. Math. Phys., 33:10 (1992), 3379-3386; Y. Brihaye, C. Gonera, S. Giller, P. Kosinski, Galilean invariance in $2+1$ dimensions, arXiv: hep-th/9503046.

[12] D. Xiao, M.-C. Chang, Q. Niu, Rev. Mod. Phys., 82:3 (2010), 1959-2007, arXiv: 0907.2021.

[13] C. Duval, Z. Horváth, P. A. Horváthy, L. Martina, P. C. Stichel, Mod. Phys. Lett. B, 20:7 (2006), 373-378, arXiv: cond-mat/0506051; Phys. Rev. Lett., 96:9 (2006), 099701, 1 pp., arXiv: cond-mat/0509806.

[14] R. Jackiw, V.P. Nair, Phys. Lett. B, 480:1-2 (2000), 237-238, arXiv: hep-th/0003130; C. Duval, P. A. Horváthy, Phys. Lett. B, 547:3-4 (2002), 306-312, arXiv: hep-th/0209166.

[15] Л. Мартина, ТМФ, 167:3 (2011), 484-495, arXiv: 1011.3545.

[16] K. Yu. Bliokh, Phys. Lett. A, 351:3 (2006), 123-124, arXiv: cond-mat/0507499.

[17] A. Bérard, H. Mohrbach, Phys. Rev. D, 69:12 (2004), 127701, 4 pp.; P. Gosselin, F. Ménas, A. Bérard, H. Mohrbach, Europhys. Lett., 76:4 (2006), 651-656, arXiv: cond-mat/0601472. 
[18] M.S. Plyushchay, Phys. Lett. B, 273:3 (1991), 250-254; 262:1 (1991), 71-78; P. A. Horváthy, M.S. Plyushchay, JHEP, 06 (2002), 033, 11 pp., arXiv: hep-th/0201228; P. A. Horváthy, M. S. Plyushchay, M. Valenzuela, Ann. Phys., 325:9 (2010), 1931-1975, arXiv: 1001.0274 .

[19] J. Lukierski, P. C. Stichel, W. J. Zakrzewski, Ann. Phys., 306:1 (2003), 78-95, arXiv: hep-th/0207149.

[20] J. F. Cariñena, J. M. Gracia-Bondia, F. Lizzi, G. Marmo, P. Vitale, Mathematical Physics and Field Theory, Julio Abad, In Memoriam, eds. M. Asorey, J. V. Garcia Esteve, M.F. Ranada, J. Sesma, Prensas Universitaria de Zaragoza, Zaragoza, 2009, arXiv: 0912.2188 .

[21] Z. Fang, N. Nagaosa, K.S. Takahashi, A. Asamitsu, R. Mathieu, T. Ogasawara, H. Yamada, M. Kawasaki, Y. Tokura, K. Terakura, Science, 302:5642 (2003), 920095, arXiv: cond-mat/0310232.

[22] S. Murakami, N. Nagaosa, S.-C. Zhang, Science, 301:5638 (2003), 1348-1351, arXiv: cond-mat/0308167.

[23] P. A. Horváthy, Phys. Lett. A, 359:6 (2006), 705-706, arXiv: cond-mat/0606472.

[24] D. J. P. Morris, D. A. Tennant, S. A. Grigera, B. Klemke, C. Castelnovo, R. Moessner, C. Czternasty, M. Meissner, K. C. Rule, J.-U. Hoffmann, K. Kiefer, S. Gerischer, D. Slobinsky, R. S. Perry, Science, 326:5951 (2009), 411-414, arXiv: 1011.1174.

[25] X.-L. Qi, R. Li, J. Zang, S.-C. Zhang, Science, 323:5918 (2009), 1184-1187, arXiv: 0811.1303.

[26] P. M. Zhang, P. A. Horváthy, J.-P. Ngome, Phys. Lett. A, 374:42 (2010), 4275-4278, arXiv: 1006.1861.

[27] J. L. Cortés, M.S. Plyushchay, Internat. J. Modern Phys. A, 11:18 (1996), 3331-3362; M. S. Plyushchay, Nucl. Phys. B, 589:1-2 (2000), 413-439, arXiv: hep-th/0004032. 